\title{
A rare loss-of-function variant of ADAM17 is associated with late- onset familial Alzheimer disease
}

\author{
Daniela Hartl ${ }^{1}$ Patrick May $\mathbb{1}^{2,3} \cdot$ Wei Gu ${ }^{1,2}$ - Manuel Mayhaus ${ }^{1}$ - Sabrina Pichler ${ }^{1}$. Christian Spaniol ${ }^{1}$. \\ Enrico Glaab ${ }^{2}$. Dheeraj Reddy Bobbili ${ }^{2}$. Paul Antony ${ }^{2}$ - Sandra Koegelsberger ${ }^{2}$ - Alexander Kurz ${ }^{4} \cdot$ Timo Grimmer $^{4}$. \\ Kevin Morgan ${ }^{5}{ }^{5}$ Badri N. Vardarajan ${ }^{6,7,8} \cdot$ Christiane Reitz $^{6,7,8,9} \cdot$ John Hardy $^{10} \cdot$ Jose Bras $^{10,11,12}$. \\ Rita Guerreiro ${ }^{10,11,12} \cdot$ Rudi Balling ${ }^{2} \cdot$ Jochen G. Schneider ${ }^{1,2} \cdot$ Matthias Riemenschneider $^{1}$ AESG
}

Received: 16 February 2018 / Revised: 5 April 2018 / Accepted: 11 April 2018 / Published online: 9 July 2018

(c) The Author(s) 2018. This article is published with open access

\begin{abstract}
Common variants of about 20 genes contributing to AD risk have so far been identified through genome-wide association studies (GWAS). However, there is still a large proportion of heritability that might be explained by rare but functionally important variants. One of the so far identified genes with rare $\mathrm{AD}$ causing variants is ADAM10. Using whole-genome sequencing we now identified a single rare nonsynonymous variant (SNV) rs142946965 [p.R215I] in ADAM17 cosegregating with an autosomal-dominant pattern of late-onset AD in one family. Subsequent genotyping and analysis of available whole-exome sequencing data of additional case/control samples from Germany, UK, and USA identified five variant carriers among $\mathrm{AD}$ patients only. The mutation inhibits pro-protein cleavage and the formation of the active enzyme, thus leading to loss-of-function of ADAM17 alpha-secretase. Further, we identified a strong negative correlation between $A D A M 17$ and APP gene expression in human brain and present in vitro evidence that ADAM17 negatively controls the expression of $A P P$. As a consequence, p.R215I mutation of ADAM17 leads to elevated AB formation in vitro. Together our data supports a causative association of the identified ADAM17 variant in the pathogenesis of AD.
\end{abstract}

Members of AESG are listed below Acknowledgements

These authors are equally contributed: Daniela Hartl, Patrick May, Wei Gu

Electronic supplementary material The online version of this article (https://doi.org/10.1038/s41380-018-0091-8) contains supplementary material, which is available to authorized users.

Daniela Hartl

daniela.hartl@uks.eu

1 Department of Psychiatry and Psychotherapy, Saarland University Hospital, Saarland University, Homburg, Germany

2 Luxembourg Centre for Systems Biomedicine (LCSB), University of Luxembourg, Esch-sur-Alzette, Luxembourg

3 Institute for Systems Biology, Seattle, WA, USA

4 Department of Psychiatry and Psychotherapy, Klinikum Rechts der Isar, TU-Muenchen, Munich, Germany

5 School of Life Sciences, Queens Medical Centre, University of Nottingham, Nottingham, UK

6 Taub Institute for Research on Alzheimer's Disease and the Aging Brain, College of Physicians and Surgeons, Columbia University,

\section{Introduction}

The central theory on Alzheimer disease (AD) pathogenesis is the amyloid cascade hypothesis with amyloid $B$ peptides $(\mathrm{A} \beta)$, formed from the $\beta$-amyloid precursor protein (APP) as etiologic agent [1]. The importance of APP metabolism was confirmed by genome-wide association studies (GWAS) and reports of rare gene variants in patients with familial

New York City, NY, USA

7 Department of Neurology, College of Physicians and Surgeons, Columbia University, New York City, NY, USA

8 Gertrude H. Sergievsky Center, College of Physicians and Surgeons, Columbia University, New York City, NY, USA

9 Department of Epidemiology, Mailman School of Public Health, Columbia University, New York City, NY, USA

10 Department of Molecular Neuroscience, Institute of Neurology, University College London, London, UK

11 UK Dementia Research Institute at UCL (UK DRI), London, UK

12 Department of Medical Sciences, Institute of BiomedicineiBiMED, University of Aveiro, Aveiro, Portugal 
AD [2]. Historically, the majority of mechanistic studies was focused on APP processing by alpha-, beta-, and gamma-secretase to illuminate the cellular pathways of $A B$ production as pharmaceutical targets [1]. In addition to APP metabolism, the so far known AD risk genes are involved in regulation of the immune system, cholesterol metabolism, cytoskeleton development, and other. However, there is still a large proportion of missing heritability that might be explained by rare but functionally important variants.

We now report a rare single-nucleotide variation (SNV) of the APP alpha-secretase ADAM17 in a family with autosomal-dominant late-onset $\mathrm{AD}$ that results in loss of enzyme function. We further identified a strong negative regulatory effect of ADAM17 on APP expression in human brain. This novel function in the newly identified $A D A M 17$ variant results in an upregulation of $A P P$ gene expression and consequently elevated $A B$ formation in vitro.

ADAM17, also known as tumor necrosis factor-alpha converting enzyme (TACE) is a sheddase with over 80 so far described substrates acting in different physiological processes [3]. It was initially described as the enzyme cleaving membrane bound tumor necrosis factor (TNF)-alpha precursor to a soluble form [4]. Later on, ADAM17 was identified as the first alpha-secretase candidate processing APP [5], followed by ADAM9 and ADAM10 [6, 7]. Rare variants of $A D A M 10$ have previously been identified in lateonset AD patients [8].

Recently, ADAM17 was also identified as main sheddase of TREM2. Rare genetic variants of TREM2 considerably contribute to $\mathrm{AD}$ risk [2].

\section{Methods}

\section{Clinical characterization of the AD family}

All participants gave written informed consent for the clinical evaluation and genetic analysis of leukocyte DNA. Clinical phenotyping, whole-genome sequencing (WGS) and genetic analysis was approved by the ethical review committee of the Bavarian medical association and by the Ethics Review Panel of the University of Luxembourg. The pedigree chart is given in Fig. 1.

The four affected patients with $\mathrm{AD}$ (patients 200, 102, 121, and 124) had a reported age of onset of 60 (patient 200), 69 (patient 102), their $A P O E$ status was $\varepsilon 3 / 4, \varepsilon 3 / 4, \varepsilon 3 / 3$, and $\varepsilon 3 /$ 3 , respectively. For patients 121 and 124, age of disease onset was not reported, age of examination at clinic was 87 and 70, respectively. Among patients unaffected by $\mathrm{AD}$, the patients 122 and 202 had symptoms of depression; and patient 123 was diagnosed with Parkinson's disease. Patient 200 was diagnosed with $\mathrm{AD}$ one year after onset of symptoms with a MMSE score of 28/30. Psychometric testing (CERAD-NAB)

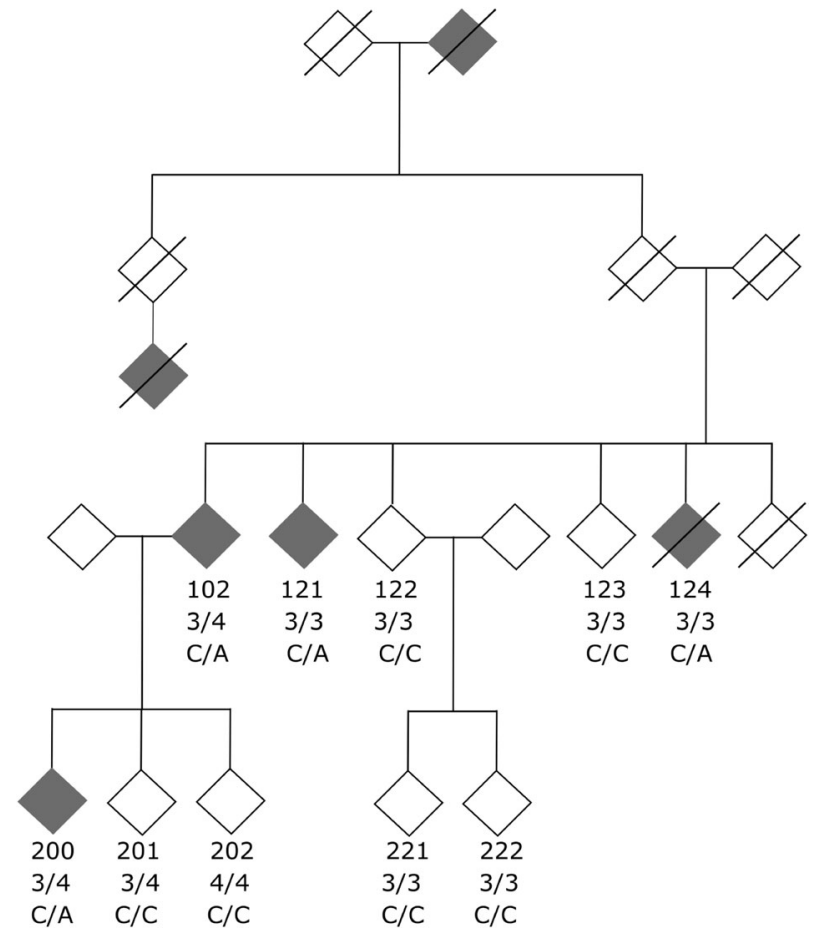

Fig. 1 Pedigree of AD family. The affected and unaffected individuals are filled in gray and white, respectively. The $A P O E$ status is given beneath individual identifiers. Carriers of the heterozygous variant (rs142946965) are indicated as C/A and carriers of the wild-type $A D A M 17$ version are indicated as $\mathrm{C} / \mathrm{C}$

revealed impairment in verbal learning and recognition as well as in delayed verbal and non-verbal recall. Cranial MRI was without abnormalities, in particular no atrophy, FDGPET showed markedly reduced tracer uptake temporal/ parietal, and occipital (sparing of the primary visual cortex) and posterior cingulate Gyrus (right $>$ left). One year after first examination clinical and imaging data showed a considerable progression, MMSE dropped to 26/30 and CERAD-NAB showed impairment in verbal fluency, verbal learning and recognition, verbal and non-verbal delayed recall, and visuoconstruction; cranial MRI revealed cortical atrophy temporal and parietal as well as hippocampal atrophy (right > left). The patient died 10 years after onset of disease symptoms. No concomitant diseases, drug abuse, or medications were reported. Neurological examination was normal and revealed no parkinsonian symptoms. Family members 201, 202, 221, and 222 were unaffected, but too young for a final diagnosis at the time of consulting with an age of examination at clinic of $55,49,55$, and 52 , respectively. The two non-affected individuals (122 and 123) had an age of examination at clinic of 81 and 74, respectively. Blood samples were taken from ten family members. DNA was extracted from leukocytes using standard procedures. Affected family members were pre-screened and negative for mutations in APP, PSEN1, and PSEN2. 


\section{Genome sequencing and analysis}

Whole-genome sequencing (WGS) was performed by Complete Genomics Inc. (CG, Mountain View, CA, USA) using paired-end, nanoarray-based sequencing-by-ligation technology [45]. Six individuals of the family were selected for WGS (Fig. 1 and Suppl. Figure 2B), two affected (102 and 200) and four unaffected $(122,123,201$, and 202) family members. After quality control, DNA samples were sent to CG for sequencing. The sequencing data was processed using the Standard Sequencing Service pipeline version 2.0. Sequencing reads were mapped against NCBI Build 37.

\section{WGS variant prioritization pipeline}

Only single-nucleotide polymorphisms (SNPs) and small indels (insertions, deletions and block substitutions up to a size of about $50 \mathrm{nt}$ ) were used for further analyses. As input for our WGS family analysis pipeline [9] we first combined all variants from the genomes of the sequenced family members into the union of variants using the CG Analysis ${ }^{\mathrm{TM}}$ Tools (CGATOOLS v.1.5) listvariant command and the CG 'var' files as input. We used CGATOOLS testvariant command to test each genome for the presence of each allele at every variant position from the union set of variants. The average fully called genome fraction, defined as the fraction of reference bases, for which alleles could be called, was $96.4 \%$, of which $99.1 \%$ were covered at least $\times 5$ and $74.9 \%$ with at least $\times 30$. On average, $99.6 \%$ of exons as defined by NCBI RefSeq 37.2 were fully called with at least $\times 5$ and $86 \%$ with at least $\times 30$. A statistical summary for all samples is given in Suppl. Table 3. We detected on average 3,441,899 SNVs and 606,446 indels and substitutions per genome. The fraction of novel variants, here defined as variants not previously found in dbSNP build 135, was on average 0.03 for SNPs and 0.27 for indels and substitutions. Relationship estimation [10] confirmed all relationships given the original pedigree information. We removed variants that were not called as high-quality calls (VQHIGH) in at least one individual. We used ISCA version 0.1.9 [11] to search for shared haplotype blocks between the two cases in the families. The shared regions are shown in Suppl. Figure 1. Variants were then filtered for the shared haplotype regions. By applying a dominant inheritance model, we filtered for variants that were called heterozygous (or have nocalls) in cases and were not called in any control genome. The remaining variants were annotated using ANNOVAR [12] (version 2015Mar12) using the NCBI RefSeq release 60 and the Ensembl release 74 genome annotations and multiple annotation databases available from the ANNOVAR webpage. We further filtered for exonic regions and excluded synonymous variants. Candidate variants should be rare in the general population (here defined by an allele frequency equal or $<1 \%$ ). Allele frequencies were determined from the European American population of the 1000 genomes project, the European NHLBI ESP exomes, and the Non-Finnish European population from the ExAC project as well as in the control data set CG69 provided by CG. Since certain genes, like mucins and olfactory receptors, are known to have higher mutation rates, variants in such genes were excluded. The list of commonly mutated genes (CMDGenes) is given in Suppl. Table 4. To narrow down the list of candidate variants, we filtered for genes that are known to be expressed in brain tissues. The brainexpressed genes were defined using the data set curated by [13], and genes expressed in brain regions from the Human BodyMap 2.0 project processed with the GeneAtlas tool (http://caballero.github.io/GeneAtlas/). We further filtered for pathogenicity by considering either loss-offunction variants (indels, stop gain, and splice-site variants) or nonsynonymous mutations predicted to be deleterious by at least 5 out of 7 pathogenicity scores (SIFT, Polyphen2_HDIV, Polyphen2_HVAR, LRT, Mutationtaster, PROVEAN, MetaSVM, fathmm-MK_coding_pred, as given by dbNSFP3.0a [14]) and two out of four conservation scores (DAMN, CADD, GERP, SiPHY). A list of candidate genes related to $\mathrm{AD}$ and neurodegenerative diseases were collected from various GWAS studies in dbGAP, the Alzgene database [15] and the Genotator tool [16]. In total, we collected a final list of 2352 candidate genes related to neurodegenerative diseases (Suppl. Table 5, NDGenes). The variants were examined using the candidate gene list and the KGGseq tool [17]. Each variant was tested if the affected gene co-occurred with any candidate gene within a certain pathway or if it was connected within a first neighborhood to one of the neurodegenerative candidate genes within a protein-protein interaction network using the STRING database [18]. The whole filtering strategy is shown in Suppl. Figure 2A.

\section{Genetic and linkage analysis}

For linkage analysis, high-quality SNP positions (complete call rate over all individuals (VQHIGH status in CG var files) were extracted from the WGS data. Using variants in linkage disequilibrium (LD) for the linkage analysis might lead to false positive results. Hence, the variants with high LD were removed by using PLINK 1.9 [19]. Further thinning of variants was performed by using mapthin software [20]. A set of 2000 variants per chromosome along with the identified variants segregating with the disease through the pedigree were used to check for genotype errors and Mendelian inconsistencies using MERLIN [21] and were subsequently removed if they were identified as errors. Remaining variants were used for linkage analysis and their genomic positions were linear interpolated based on hapmap genetic map (2011- 
01_phaseII_B37). MERLIN was used to perform both haplotyping and multipoint parametric linkage analysis with a rare dominant disease model with a disease frequency of 0.0001 and penetrance of $0.0001,1.0,1.0$. Haplotyping results were visualized using Haplopainter [22] (Suppl. Figure 2BC). Using the R package 'paramlink' [23] we calculated the power of the pedigree given as the maximal LOD score (1.20412) under an autosomal-dominant inheritance model and 10,000 simulated markers. Relationship detection between all individuals was performed using the software GRAB [10]. Validation of results by Sanger sequencing was carried out by Seq IT GmbH, Kaiserslautern, Germany.

\section{Expression correlation analysis of ADAM17 and APP in human brain tissue}

Correlation analysis of APP and ADAM17 gene expression was carried out using a sample set obtained from fresh frozen post-mortem prefrontal cortex of 201 AD samples and 83 cognitively healthy controls, provided by The Netherland Brain Bank (NBB). A second sample set used for independent validation of results contained 57 fresh frozen post-mortem human temporal cortex samples (33 AD samples and 24 cognitively healthy controls) provided by Brain Bank Munich (MUC). Full sample characteristics are given in Suppl. Table 2. Total RNA was extracted from human brain tissue using either miRNeasy Mini Kit or RNeasy Lipid Tissue Mini Kit (Qiagen) according to manufacturer's protocol including on-column DNA digestion with RNase free DNase Set (Qiagen). Total RNA was amplified and labeled with biotin using Ambion ${ }^{\circledR}$ Illumina ${ }^{\circledR}$ TotalPrep ${ }^{\mathrm{TM}}$ RNA Amplification Kit (Ambion) according to manufacturer's protocol. cRNA was eluted in $50 \mu \mathrm{l}$ nuclease-free water and precipitated using ammonium acetate $\left(\mathrm{NH}_{4} \mathrm{OAc}\right)$ when concentration was $<150 \mathrm{ng} / \mu \mathrm{l}$. Direct hybridization of $750 \mathrm{ng}$ biotin labeled cRNA to Illumina ${ }^{\circledR}$ Sentrix BeadChips HumanHT-12 v4 (Illumina) was conducted according to manufacturer's protocol. Whole-genome gene expression analysis incorporated 47,231 markers per sample including coding as well as noncoding transcripts based on Human RefSeq 38 (NCBI RefSeq database) and supplementary UniGene content. Processed BeadChips were scanned using the HiScan (Illumina). In the first quality check step, background subtraction, and quantile normalization were executed with GenomeBead Studio Module for gene expression. Markers with a detection $p$-value $\geq 0.05$ were considered as no-call. Call rates for each marker were calculated separately in both groups (AD vs. control). Markers with a call rate $<90 \%$ in both groups were considered as non-informative and were excluded from further statistical analysis. Signal intensities were $\log _{2}$ transformed for further analysis. Correlation between the expression of $A D A M 17$ and $A P P$ was tested using the linear model fit function $(\mathrm{lm})$ in the $\mathrm{R}$ package. $p$ value of the coefficient (slope of the fit) and adjusted $R^{2}$ were reported. Correlation between ADAM17 and ADAM17 target gene expression was tested using the correlation test in $\mathrm{R}$.

Additionally, the MERAV database and analysis tool [30] was used to examine gene expression correlations between ADAM17 and APP in non-brain and brain tissues from public databases like ArrayExpress, GEO and the human body index. MERAV is a tool for comparing gene expression across different human tissues and cell types. The quantile normalized log-transformed expression values for the four genes for normal non-brain (502) and normal brain tissues (186) were downloaded and Pearson correlation between gene expression for all gene pairs were calculated using tool cor.test in R. Additionally, we tested the significance of the difference between correlations coefficients obtained for the non-brain and brain tissues using the R library psych and the one-sided paired $r$ test.

\section{Generation of ADAM17 variants}

ADAM17 cDNA was derived from clone HsCD00342307 (Harvard Medical School). Wild-type ADAM17 was amplified and digested with KpnI and BamHI primers (for primer sequences see Suppl. Table 7), and ligated into pCDNA3.1 (+) (Invitrogen \# V790-20). Site-directed mutagenesis of ADAM17 was done via PCR-driven overlap extension (PMID 17446874). For p.R215I and p.E406A mutagenesis primer sequences are given in Suppl. Table 6. Hybridization of 5' and $3^{\prime}$ portions of $A D A M 17$ variants was used for the amplification of full length $A D A M 17$ variants. For subcloning into pcDNA3.1(+) the full length $A D A M 17$ variants were enriched via PCR amplification using the primers KpnI_f and BamHI_r. Digestion and ligation steps were done as described above for wild-type ADAM17. Bacterial cultures with $A D A M 17$ constructs were grown at $24^{\circ} \mathrm{C}$ for $48 \mathrm{~h}$.

\section{Cell culture}

SH-SY5Y cells stably expressing human $\mathrm{APP}_{695}$ carrying the Swedish double mutation (in pcDNA3.1, selection with $0.3 \mathrm{mg} / \mathrm{ml}$ hygromycin) were kindly provided by Tobias Hartmann and Marcus Grimm (Saarland University). SHSY5Y cells were stably transfected with expression constructs for $A D A M 17$ variants (in pcDNA3.1, selection with $500 \mu \mathrm{g} / \mathrm{ml}$ geneticin). Cells were grown in DMEM with selection antibiotics and 10\% fetal calf serum (FCS). For rescue experiments, cells were supplied with media containing $100 \mathrm{nM}$ TAPI-0 (SantaCruz) or equal volume of DMSO as control. Medium was changed every second day, cells were checked for mycoplasma contamination. 


\section{Protein and gene expression analyses in cells}

The amounts of ADAM17 and APP in cellular lysates were determined by standard Western blotting techniques. Antibodies directed against ADAM17 or APP were obtained from commercial suppliers (ADAM17/TACE C15 and H300, SantaCruz; APP 6E10, Covance). For lysis, cells were washed with ice-cold PBS and resuspended in ice-cold standard RIPA buffer containing $10 \mathrm{mM} 1,10$ phenantroline (Sigma-Aldrich) and protease inhibitor cocktail (Complete, Roche). Blots were developed using the SuperSignal West Femto Chemiluminescent Substrate (Pierce) and analyzed using the Peqlab FUSION XPress Imaging System. BetaActin or Alpha-Tubulin was analyzed as loading control. For statistical analysis, signals were normalized to loading controls and statistical significance was determined by nonparametric Kruskal-Wallis test and Dunn's multiple comparison test using Prism software (version 6.0d; Graphpad). Multiplicity adjusted $p$-values are given. Only relative signals (to control) were combined from different blots and all samples represent biological replicates.

Secreted $A \beta_{40}$ and $A \beta_{42}$ was quantified in cell culture media using commercial ELISA kits (EZBRAIN $A \beta_{40}$ and $\mathrm{A} \beta_{42}$ Brain ELISA Kit, respectively Millipore). For ELISA, culture media was removed, cells were washed with PBS, then incubated in fresh medium containing $1 \%$ FCS for $48 \mathrm{~h}$. After incubation, medium was collected, spun down and supernatant was used for measurements. Statistical significance of data was determined by ANOVA with Fisher's LSD test for pairwise comparisons using Prism software (version 6.0d; Graphpad).

Total RNA from cells was prepared using miRNeasy Mini Kit (Qiagen) according to manufacturer's protocol. High-Capacity cDNA Reverse Transcription Kit (Applied Biosystems) was used for cDNA synthesis. Levels of APP mRNA expressed in SH-SY5Y cells were determined by quantitative RT-PCR using the Applied Biosystems 7500 Fast Real-Time PCR System. Samples were measured in triplicates, and calculations were performed using the Prism software (Graphpad, version 6.0d). Values for APP were normalized to $18 \mathrm{~S}$ expression determined in parallel in the respective samples. Fold changes were calculated by applying the $2^{\wedge}$-ddCT method.

\section{Results}

\section{Identification of an ADAM17 variant associated with familial AD}

We identified an SNV of ADAM17 co-segregating with the disease in a family with an autosomal-dominant pattern of inheritance of late-onset AD. Affected family members lacked pathogenic mutations of known AD genes. Wholegenomes of six family members (two affected and four unaffected, Fig. 1) were sequenced and analyzed by prioritizing rare, potential damaging variants that segregated with the disease. We searched for segregating variants by applying an autosomal-dominant inheritance model. In total 7,009,082 variants (SNPs and indels) different from the reference genome were identified in at least one family member. After strict quality filtering, 6.2 million variants remained. Due to the pedigree structure, we filtered for shared haplotype regions (Suppl. Figures 1 and 2A) between the two affected individuals $(n=3,903,193)$ and autosomal-dominant mode of inheritance $(n=187,733)$ filtering for heterozygous variants in the two affected and homozygous wild-type genotypes in the unaffected individuals 122 and 123. To further narrow down the list of variants, we screened for rare variants $(n=18,597)$ with predicted exonic defects $(n=64)$. After filtering out commonly mutated $(n=51)$ and variants in genes that are not brain-expressed $(n=25)$, we prioritized the remaining variants according to their predicted pathogenicity and conservation ( $n=11$, Suppl. Figure 2A, Suppl. Table 1). Only two out of the nine candidate variants were known relevant candidate genes (ADAMI7 and ADRA2A). Additionally, we performed linkage analysis for the pedigree using MERLIN [21]. Only two variants (ADAM17 and $D D H D 2)$ fall into a region that showed the maximal LOD score (1.20) for this pedigree as calculated with 'paramlink' [23] (Suppl. Figure 2B-C). The ADAM17 variant (NM_003183:exon6:c.G644T:p.R215I; rs142946965) was the only variant in an $\mathrm{AD}$ relevant gene predicted pathogenic by 7 out of 8 prediction programs (SIFT, Polyphen (HDIV/HVAR)), LRT, MutationTaster, Provean and FATHMM), conserved by three out of four conservation scores (DAMN, GERP, SiPHy) and additionally located within the maximal LOD score region of the pedigree. The presence of the heterozygous ADAMI7 variant (rs142946965) in the two affected family members was confirmed by Sanger sequencing and identified in two additional affected family members $(121,124$, Fig. 1) that have not been analyzed by whole-genome sequencing (WGS). Also, the absence of the variant was verified in the four non-affected family members and in the non-affected family members 221 and 222 (Fig. 1) that have not been analyzed by WGS. The information generated by Sanger sequencing of additional family members results in a maximal LOD score of 1.81 as calculated with 'paramlink'.

Together, our genetic approach including WGS, confirmation, and extension by Sanger sequencing identified the SNV rs142946965 of ADAM17 to co-segregate with AD cases among the analyzed family members.

To further explore the possible relevance of the rs142946965 variant in AD we genotyped a German cohort 
$(N=1045: 969$ cases, 76 controls $)$ and analyzed this variant in exome sequencing data from a UK cohort $(N=571: 331$ cases, 240 controls), and a cohort from the USA (Washington Heights-Hamilton Heights-Inwood Community Aging Project; WHICAP; $N=3834: 1567$ cases, 2267 controls). The variant was not present within the control subjects. The same was true for an additional cohort of 468 unrelated Caucasian individuals of age 60 or higher from several UK and US brain banks who did not have a neurodegenerative disease diagnosis or disease-associated neuropathology at the time of death (Healthy exomes database, version 1.0) [24]. We identified five additional heterozygous variant carriers among $\mathrm{AD}$ subjects, one in each of the German and UK cohort, and three in the US cohort. The German variant carrier had an age of disease onset of 79 and the APOE status was $\varepsilon 2 / 3$. The British variant carrier had an age at death of 92 , with a hypothesized onset of disease at the age of 84 years, the $A P O E$ status was $\varepsilon 3 / 4$. The three variant carriers in the US cohort had an age of disease onset of 85,85 , and 90 , and one patient carries the APOE \&4 allele. All three US variant carriers were of Hispanic ethnicity.

Subsequent screening of genetic databases revealed an ultra-rare occurrence of the heterozygous rs 142946965 variant in the European population (ESP6500_ea: 0.000077, 1000g_eur: 0.000199681, ExAC_NFE: 0.0001049) and no presence in any non-European populations. In the gnomAD database (http://gnomad.broadinstitute.org) from February 2017 the variant was present in 9 out of 138,390 individuals (allele frequency of 0.0000325), only in the heterozygous form and only present in the non-Finnish European population.

\section{The p.R215I mutation affects pro-protein convertase cleavage of ADAM17}

A disintegrin and metalloprotease family (ADAM) members are multidomain proteins synthesized as latent precursors that are converted to mature, active enzymes by pro-protein convertase [25]. Enzymatic activity is inhibited via a cysteine switch mechanism forming a complex between a cysteine residue within the pro-domain and the zinc ion in the active center of the catalytic domain [26]. Activation of ADAMs requires proteolytic cleavage of the pro-domain. Pro-ADAM17 is activated in the trans-Golgi-network through furin cleavage at the last four amino acids preceding the catalytic domain [27]. The identified rs142946965, R215I variant of $A D A M 17$ causes a missense mutation changing positively charged, polar arginine to the non-polar, hydrophobic amino acid, isoleucine. The change is located at position 215 (corresponding to the canonical sequence of human ADAM17), right next to the pro-protein convertase cleavage motif (spanning positions 210-214 [28]), suggesting a possible interference with pro-protein convertase binding to ADAM17. To analyze possible effects of the mutation on ADAM17 biochemical function, we generated neuroblastoma (SH-SY5Y) cell lines stably overexpressing the newly identified variant of ADAM17 (p.R215I). In addition, we generated SH-SY5Y cells overexpressing human, wild-type ADAM17 (wild-type), and a catalytically inactive/dominant-negative form of ADAM17 (p.E406A), known from literature [29].

Over-expression of ADAM17 in cells resulted in significant upregulation of total and pro-ADAM17 protein levels as compared to the parental cell line (control). This was true for all three variants, p.R215I, p.E406A, and wildtype ADAM17 (Fig. 2a-c). There was no significant difference between the p.R215I variant, the p.E406A variant and the wild-type variant of pro-ADAM17 levels. In contrast, levels of the shorter, mature form of ADAM17 lacking

A

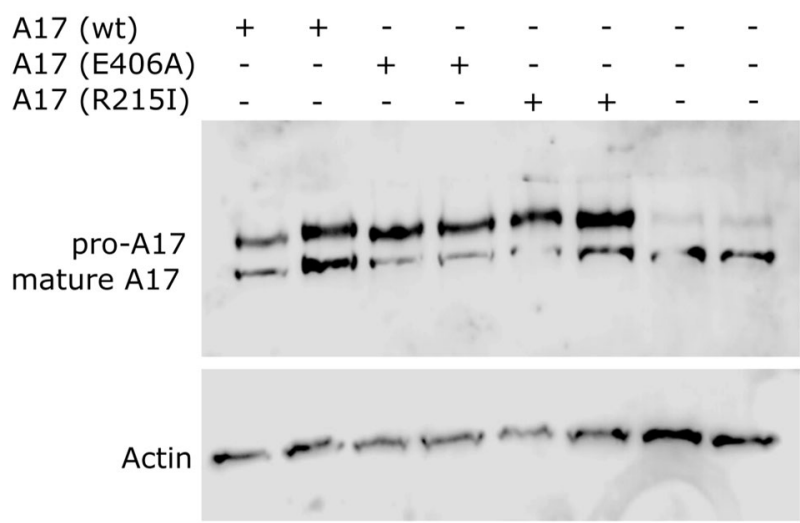

B
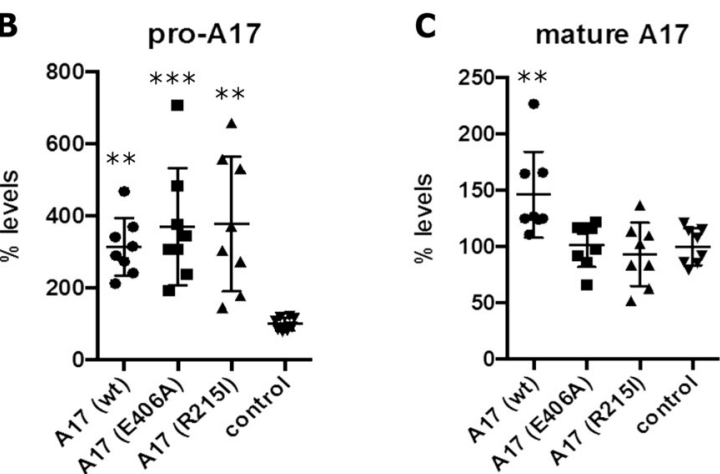

Fig. 2 The R215I variant affects maturation of ADAM17. a Western blot showing ADAM17 (A17) protein expression in SH-SY5Y cells, over-expressing wild-type (wt) ADAM17, a dominant-negative form (E406A), or the newly identified AD variant of ADAM17 (R215I). The parental cell line was analyzed as control, actin-beta was analyzed as loading control (Actin). b, c Quantification of pro-ADAM17 signals shows significant upregulation in cell lines over-expressing ADAM17 variants $(n=8 ; \mathrm{p}(\mathrm{wt})=0.0057 ; \mathrm{p}(\mathrm{E} 406 \mathrm{~A})=0.0009 ; \mathrm{p}(\mathrm{R} 215 \mathrm{I})=$ $0.0014)$, respectively, as compared to the parental cell line (control). Quantification of mature ADAM17 signals only shows significant upregulation in cell lines over-expressing wild-type ADAM17 ( $n=8$; $\mathrm{p}(\mathrm{wt})=0.0092)$, as compared to the parental cell line (control). Asterisks indicate significance. Error bars indicate mean with SD 
the pro-domain, were significantly upregulated exclusively in cells over-expressing wild-type ADAM17, but not altered in cells over-expressing p.R215I or p.E406A variants, as compared to control cells, respectively (Fig. 2a, c). These results demonstrate that as previously described elsewhere for the inactive p.E406A variant, pro-protein convertase cleavage is severely impaired in the newly identified ADAM17 variant (p.R215I), resulting in a lack of mature, catalytically active ADAM17 molecules.

\section{ADAM17 inhibits APP expression in vitro}

Three members of the ADAM family, ADAM9, ADAM10, and ADAM17, show APP alpha-secretase activities [5-7]. The alpha-secretase initiates the non-amyloidogenic pathway by cleaving APP within the beta-amyloid sequence, which precludes the formation of beta-amyloid and further generates the neurotrophic and neuroprotective soluble Nterminal fragment of APP (sAPP $\alpha$ ).

To analyze the effect of ADAM17 variants on $A B$ formation, we created SH-SY5Y cell lines over-expressing both human APP and ADAM17 (wild-type, p.E406A, or p.R215I variants, respectively). Maturation of mutated ADAM17 was again reduced in these cells (data not shown).

We first measured total APP protein and gene expression in ADAM17 and APP transgenic SH-SY5Y cell lines. Surprisingly, APP protein and gene expression were significantly reduced in cells expressing wild-type ADAM17 as compared to control cells expressing mutated forms of ADAM17, or APP only (Fig. 3a-c). Downregulation of APP gene expression in cells expressing wild-type ADAM17 was also observed in cells expressing endogenous APP (Suppl. Figure 3). In accordance with the observed reduction of APP expression by wild-type ADAM17, Aß40, and $\mathrm{A} ß 42$ secretion as measured by ELISA was upregulated in cells expressing the R215I variant as compared to cells expressing wild-type ADAM17 (Fig. 3d, e).

To analyze whether the observed downregulation of APP expression could be rescued by ADAM17 inhibition, we treated SH-SY5Y cells expressing both, APP and wild-type ADAM17, with a metalloprotease inhibitor (TAPI-0). Treatment of cells with $100 \mathrm{nM}$ TAPI-0 inhibitor for $16 \mathrm{~h}$ had no significant effect on APP expression, but long-term treatment (6 days) with regular medium change resulted in full rescue of APP gene and protein expression, suggesting an active functional role of ADAM17 in regulation of APP expression (Fig. 3a-c).

\section{ADAM17 is negatively correlated with APP expression in human brain}

We next analyzed a possible correlation between $A P P$ and $A D A M 17$ gene expression in human brain. Analysis of gene expression data from 284 human prefrontal cortex samples (NBB data: 201 AD patients and 83 controls, Suppl. Table 2) showed a very strong negative correlation $\left(p=2.2 \times 10^{-16}, r^{2}=\right.$ 0.55 for the average of APP markers ILMN_2404065, ILMN_2404063, and ILMN_1653283 compared to the average of ADAM17 markers ILMN_1765779 and ILMN_2121068) of $A D A M 17$ and APP mRNA both, in $\mathrm{AD}$ and control brains (Fig. 4a). This negative correlation was replicated in an independent sample comprising 57 human temporal cortex samples (MUC data: $33 \mathrm{AD}$ patients and 24 controls, $p=5.8 \times 10^{-10}, r^{2}=0.44$, Suppl. Table 2; Fig. 4b). These results strongly argue for a general functional connection of both genes in human brain which is not restricted to $\mathrm{AD}$ patients.

To find out whether ADAM17 expression might be correlated with expression of its other targets as well, we used the human brain gene expression data set (NBB) to check for possible correlations of ADAM17 with 71 known ADAM17 targets. Among the analyzed ADAM17 target genes (Supplementary Table 7), ADAM17 only showed high correlation (defined as Pearson correlation coefficient of \pm 0.7 to 1$)$ with $P R N P(-0.77)$ and $A P P(-0.74)$. Other AD related targets, such as TREM2 or SORL1 only had low to moderate correlation coefficients (Suppl. Table 7).

In addition, we explored possible correlations between $A P P$ and both other alpha-secretase genes, ADAM9 and $A D A M 10$, using our human brain gene expression data sets. We observed a very weak negative correlation of ADAM9 with $A P P$ gene expression (NBB data: $p=0.06, r^{2}=0.01$; MUC data: $p=0.02, r^{2}=0.06$; for ADAM9 marker ILMN_1727524). For ADAM10, a weak positive correlation with $A P P$ expression was observed (NBB data: $p=3.3 \times$ $10^{-8}, r^{2}=0.09$; MUC data: $p=2.6 \times 10^{-4}, r^{2}=0.17$; for the average of ADAM10 markers ILMN_1718946 and ILMN_2148360).

We next evaluated possible correlations between $A P P$ and $A D A M 17$ gene expression in brain and non-brain tissues from public databases using the MERAV tool [30]. Again, a significant negative correlation was observed in brain tissues $(r=-0.27, p=0.00024)$, whereas in nonbrain tissues, no correlation between ADAM17 and APP was present $(r=0.0089, p=0.3421)$.

Together, we found that among known ADAM17 substrates, gene expression of $A P P$ and $P R N P$ showed the highest observed overall correlation with $A D A M 17$ gene expression in the brain. Both correlations were negative and $A P P$ gene expression was not significantly correlated with gene expression of $A D A M 9$ and ADAM10.

\section{Discussion}

Using WGS we identified a single rare nonsynonymous variant p.R215I in ADAM17 shared in all four affected 


\section{A}

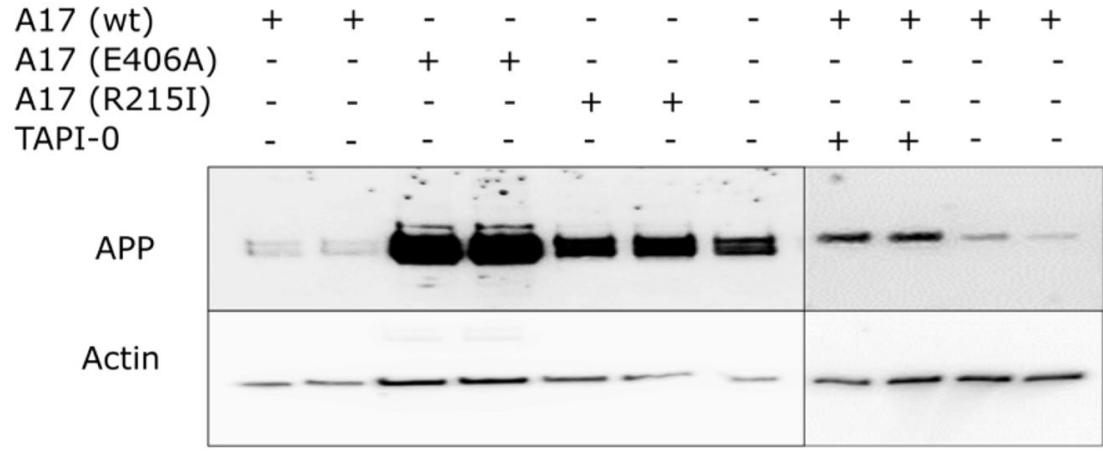

B

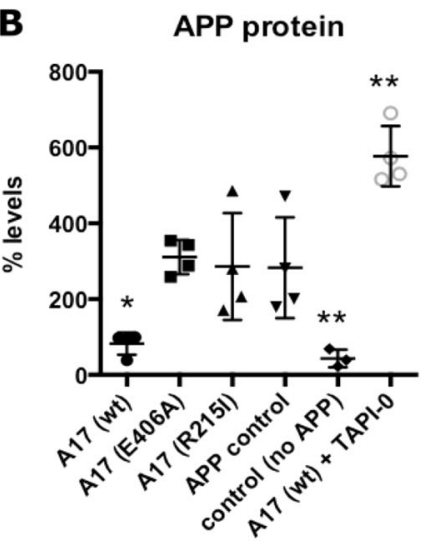

C

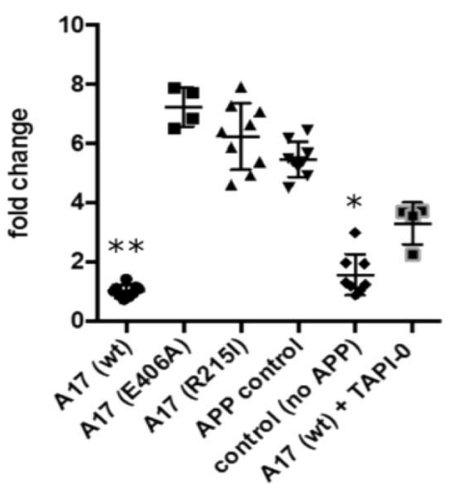

D

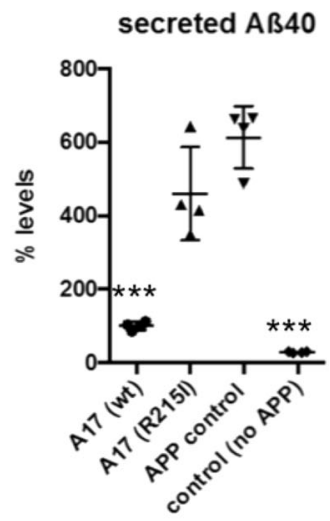

E secreted $\mathrm{AB42}$

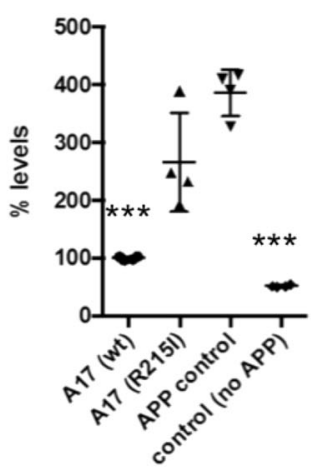

Fig. 3 APP expression is downregulated by ADAM17. a Western blot showing total APP protein expression in lysates of cells overexpressing APP and respective variants of ADAM17 (A17). b Quantification of total APP protein signals shows significant downregulation in cells over-expressing wild-type (wt) ADAM17 and APP as compared to cells only expressing APP (APP control). No significant change was observed in cells expressing ADAM17 E406A and R215I variants, respectively, as compared to APP control cells. Rescue of APP expression was observed in A17 wt cells after treatment with ADAM inhibitor TAPI-0. c APP gene expression is

individuals and co-segregating in an extended family with an autosomal-dominant pattern of late-onset AD. We cannot exclude the possibility that other co-segregating variants are involved in $\mathrm{AD}$ pathogenesis as well. However, the $A D A M 17$ variant was the only variant in a so far known $\mathrm{AD}$ relevant gene that was also predicted pathogenic by 7 out of 8 prediction programs, conserved by three out of four conservation scores and additionally located within the maximal LOD score region of the pedigree. Subsequent follow-up of this variant in genotyping and whole-exome sequencing data in 5450 additional case/control samples from Germany, the UK and the USA identified five heterozygous variant carriers among $\mathrm{AD}$ patients and none within the control subjects. Information derived from genetic population databases revealed an ultra-rare allele frequency among Europeans and absence in non-European populations. significantly downregulated in cells over-expressing A17 wt and APP as compared to APP control cells $(n=9, \mathrm{p}(\mathrm{wt})=0.001)$. Rescue of APP expression was observed in A17 wt cells after treatment with ADAM inhibitor TAPI-0 $(n=3)$. APP was also significantly downregulated in the parental cell line that did not over-express APP $(n=9$, $p=0.0341)$. d ELISA of $A \beta_{40}$ or e $A \beta_{42}$ in media of SH-SY5Y cells over-expressing APP shows significant downregulation of both $A B$ forms in $\mathrm{A} 17(\mathrm{wt}),(\mathrm{p}(\mathrm{A} ß 40)<0.0001 ; \mathrm{p}(\mathrm{A} 342))=0.0007 ; n=4)$ cells as compared to APP control cells. For $\mathbf{b}$ to e: Asterisks indicate significance. Error bars indicate mean with SD

During the functional characterization of the new ADAM17 variant, we demonstrated failure of ADAM17 maturation causing a loss of alpha-secretase function. Previously, two rare missense mutations of alpha-secretase ADAM10, a close relative of ADAM17, were identified in autosomal-dominant families with AD [8]. Interestingly, as reported here for ADAM17, these ADAM10 mutations were demonstrated to impair pro-domain cleavage as well [31]. Loss of ADAM10 function also resulted in enhanced $\mathrm{A} B$ formation in mice [31].

Focusing on the ADAM17 target APP, we identified a surprising strong negative correlation between $A D A M 17$ and $A P P$ gene expression in human brain tissue. Interestingly, a recent report addressing the strongest identified genetic risk factor for sporadic AD so far, Apolipoprotein $\mathrm{E}(A P O E)$, has uncovered a yet unknown differential effect of $A P O E 2, A P O E 3$, and APOE4 variants on APP 

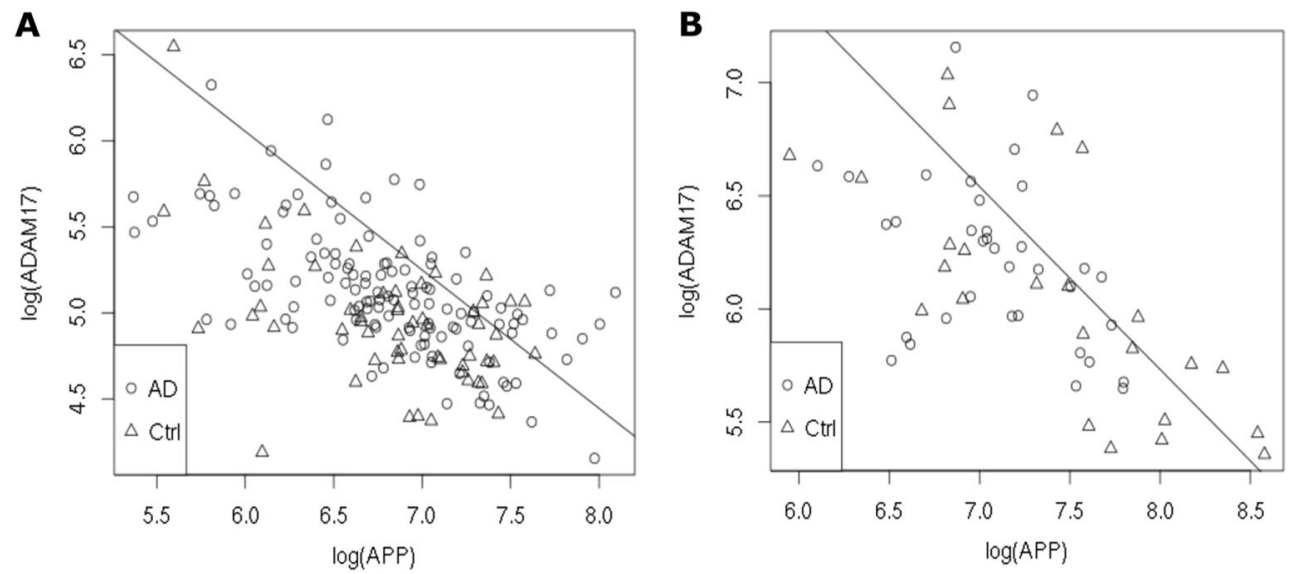

Fig. $4 A P P$ expression is negatively correlated with $A D A M 17$ expression in human brain. Average $\log _{2}$ of $A D A M 17$ gene expression against average $\log _{2}$ of $A P P$ gene expression was plotted. a NBB sample set; $\mathbf{b}$ MUC sample set

transcription [32]. These results corroborate a central involvement of APP in AD pathology and shed new light on the role of differential APP gene expression in $\mathrm{AD}$ pathogenesis.

Using cellular models, we confirmed that overexpression of wild-type ADAM17 caused a reduction of APP gene and protein expression, suggesting a novel mechanism of APP expression regulation by ADAM17. In literature, different pathways are described potentially linking ADAM17 to APP transcription. von Rotz et al. demonstrated that AICD regulates transcription of $A P P$ after being transported to the nucleus; but AICD generated through the alpha-secretase pathway is rather unstable and rapidly degraded [33, 34]. Also, cleavage of signaling receptors such as the p75 neurotrophin receptor or the TGFß-receptor by ADAM17 could potentially influence APP transcription [35-38]. However, the exact mechanism has yet to be determined.

In contrast to wild-type ADAM17, over-expression of the R215I version of ADAM17 failed to reduce APP expression and caused elevated $A B$ levels in vitro. In line with our results, it was previously reported that induction of ADAM17 coincided with downregulation of APP in rat brain and HEK293 cells, and reduced $A B$ accumulation and AD-like pathology in the Tg2576 mouse model [39-41]. Moreover, decreased ADAM17-mediated alpha-secretase activity was previously shown to promote disease progression in prion disease and $\mathrm{AD}$ and to increase accumulation of $A ß$ and pathogenic prions [42]. In cancer cells, APP was demonstrated to regulate $A D A M 17$ gene expression [43]. Together, these findings suggest that the R215I mutation of ADAM17 causes loss of alpha-secretase function and consequently elevated $A B$ production.

Given that over 80 substrates of ADAM17 have been identified so far, the newly identified variant of ADAM17 most probably causes multiple effects that might play a role in $\mathrm{AD}$ pathogenesis. For example, ADAM17 has recently been demonstrated to be the main sheddase of TREM2, a transmembrane protein that was identified as $\mathrm{AD}$ risk factor by GWAS [2, 44]. However, the exact mechanisms how ADAM17 contributes to $\mathrm{AD}$ pathogenesis remain to be analyzed in the future.

Our results corroborate an involvement of the alphasecretase ADAM17 in AD pathogenesis by identifying a novel rare loss of function variant of this gene. Future largescale genetic sequencing approaches aiming at the identification and gene-based analyses of ADAM17 variants are mandatory to fully understand the genetic contribution of ADAM17 to AD.

Acknowledgements We are grateful to the probands and their families for contributing to this study. Computational results presented in this paper were carried out using the HPC facility of the UL (http://hpc.uni. lu). We thank the ISB Family Genomics group for their support and project management of the WGS data, and Katrin Williams for excellent technical assistance. We would like to gratefully acknowledge all donors and their families for the tissue provided for this study. Human post-mortem tissue was obtained from the South West Dementia Brain Bank, London Neurodegenerative Diseases Brain Bank, Manchester Brain Bank, Newcastle Brain Tissue Resource and Oxford Brain Bank, members of the Brains for Dementia Research (BDR) Network. The BDR is jointly funded by Alzheimer's Research UK and the Alzheimer's Society in association with the Medical Research Council. We also wish to acknowledge the neuropathologists at each center and BDR Brain Bank staff for the collection and classification of the samples. Tissue provided by the Newcastle Brain Tissue Resource is funded in part by a grant from the UK Medical Research Council (G0400074), by NIHR Newcastle Biomedical Research Centre and Unit awarded to the Newcastle upon Tyne NHS Foundation Trust and Newcastle University, and by a grant from the Alzheimer's Society and Alzheimer's Research UK as part of the Brains for Dementia Research Project.

Alzheimer's disease Exome Sequencing Group Celeste Sassi ${ }^{13}$, J. Raphael Gibbs ${ }^{14}$, Dena Hernandez ${ }^{14}$, Keeley J. Brookes ${ }^{15}$, Tamar Guetta-Baranes $^{15}$, Paul T. Francis ${ }^{16}$, Michelle K. Lupton ${ }^{17}$, Kristelle Brown ${ }^{15}$, John Powell ${ }^{18} \&$ Andrew Singleton ${ }^{14}$ 
${ }^{13}$ Department of Experimental Neurology, Center for Stroke Research Berlin (CSB), Charité - Universitätsmedizin Berlin, Corporate Member of Freie Universität Berlin, Humboldt-Universität zu Berlin, and Berlin Institute of Health, Berlin, Berlin, Germany; ${ }^{14}$ Laboratory of Neurogenetics, National Institute on Aging, National Institutes of Health, Bethesda, MD, USA; ${ }^{15}$ Translation Cell Sciences-Human Genetics, School of Life Sciences, Queens Medical Centre, University of Nottingham, Nottingham, UK; ${ }^{16}$ Brains for Dementia Research Resource, Wolfson Centre for Age Related Diseases, King's College London, London, UK; ${ }^{17}$ QIMR Berghofer Medical Research Institute, Brisbane, Queensland, Australia; ${ }^{18}$ King's College London, Institute of Psychiatry, Psychology and Neuroscience, London, UK

Funding Financial support was provided by the University of Luxembourg (UL) -Institute for Systems Biology (ISB) Strategic Partnership by 'le plan Technologies de la Sante par le Gouvernment du Grand-Duche de Luxembourg' through the Luxembourg Centre for Systems Biomedicine and the UL (PM). Financial support was further given from Saarland University (Homfor2014) to DH and the German Federal Ministry of Education and Research (BMBF) National Genome Research Network (NGFN) grant No. 01GS08125 (MR), and the Helmholtz Alliance for Mental Health in an Aging Society (HELMA) Grant No. Ha-15 (MR). The exome-sequencing work was supported in part by the Alzheimer's Research UK (ARUK), by an anonymous donor, by the Wellcome Trust/MRC Joint Call in Neurodegeneration award (WT089698) to the UK Parkinson's Disease Consortium whose members are from the UCL/Institute of Neurology, the University of Sheffield and the MRC Protein Phosphorylation Unit at the University of Dundee, by the Big Lottery (to KM). The exome-sequencing component was also supported in part by the Intramural Research Programs of the National Institute on Aging and the National Institute of Neurological Disease and Stroke, National Institutes of Health, Department Of Health and Human Services Project number ZO1 AG000950-10. The work by RG and JB is supported by Research Fellowships from the Alzheimer's Society. The WHICAP study was supported by NIH grant RF1AG054023 and R01AG037212.

\section{Compliance with ethical standards}

Conflict of interest TG has reported in having received consulting fees from Actelion, Eli Lilly, MSD; Novartis, Quintiles, Roche Pharma, and lecture fees from Biogen, Lilly, Parexel, Roche Pharma, and also grants to his institution from Actelion and PreDemTech. The remaining authors declare that they have no conflict of interest.

Open Access This article is licensed under a Creative Commons Attribution 4.0 International License, which permits use, sharing, adaptation, distribution and reproduction in any medium or format, as long as you give appropriate credit to the original author(s) and the source, provide a link to the Creative Commons license, and indicate if changes were made. The images or other third party material in this article are included in the article's Creative Commons license, unless indicated otherwise in a credit line to the material. If material is not included in the article's Creative Commons license and your intended use is not permitted by statutory regulation or exceeds the permitted use, you will need to obtain permission directly from the copyright holder. To view a copy of this license, visit http://creativecommons. org/licenses/by/4.0/.

\section{References}

1. Ballard C, Gauthier S, Corbett A, Brayne C, Aarsland D, Jones E. Alzheimer's disease. Lancet. 2011;377:1019-31.
2. Cuyvers E, Sleegers K. Genetic variations underlying Alzheimer's disease: evidence from genome-wide association studies and beyond. Lancet Neurol. 2016;15:857-68.

3. Zunke F, Rose-John S. The shedding protease ADAM17: physiology and pathophysiology. Biochim Biophys Acta. 2017;1864: 2059-70.

4. Black RA, Rauch CT, Kozlosky CJ, Peschon JJ, Slack JL, Wolfson MF, et al. A metalloproteinase disintegrin that releases tumour-necrosis factor-alpha from cells. Nature. 1997;385: 729-33.

5. Buxbaum JD, Liu KN, Luo Y, Slack JL, Stocking KL, Peschon $\mathrm{JJ}$, et al. Evidence that tumor necrosis factor alpha converting enzyme is involved in regulated alpha-secretase cleavage of the Alzheimer amyloid protein precursor. J Biol Chem. 1998;273: 27765-7.

6. Koike H, Tomioka S, Sorimachi H, Saido TC, Maruyama K, Okuyama A, et al. Membrane-anchored metalloprotease MDC9 has an alpha-secretase activity responsible for processing the amyloid precursor protein. Biochem J. 1999;343(Pt 2):371-5.

7. Lammich S, Kojro E, Postina R, Gilbert S, Pfeiffer R, Jasionowski $\mathrm{M}$, et al. Constitutive and regulated alpha-secretase cleavage of Alzheimer's amyloid precursor protein by a disintegrin metalloprotease. Proc Natl Acad Sci USA. 1999;96:3922-7.

8. Kim M, Suh J, Romano D, Truong MH, Mullin K, Hooli B, et al. Potential late-onset Alzheimer's disease-associated mutations in the ADAM10 gene attenuate $\alpha$-secretase activity. Hum Mol Genet. 2009;18:3987-96.

9. Schubert J, Siekierska A, Langlois M, May P, Huneau C, Becker $\mathrm{F}$, et al. Mutations in STX1B, encoding a presynaptic protein, cause fever-associated epilepsy syndromes. Nat Genet. 2014;46:1327-32.

10. Li H, Glusman G, Huff C, Caballero J, Roach JC. Accurate and robust prediction of genetic relationship from whole-genome sequences. PLoS ONE. 2014;9:e85437.

11. Roach JC, Glusman G, Smit AFA, Huff CD, Hubley R, Shannon PT, et al. Analysis of genetic inheritance in a family quartet by whole-genome sequencing. Science. 2010;328:636-9.

12. Wang K, Li M, Hakonarson H. ANNOVAR: functional annotation of genetic variants from high-throughput sequencing data. Nucleic Acids Res. 2010;38:e164.

13. Pinto D, Delaby E, Merico D, Barbosa M, Merikangas A, Klei L, et al. Convergence of genes and cellular pathways dysregulated in autism spectrum disorders. Am J Hum Genet. 2014;94:677-94.

14. Liu X, Wu C, Li C, Boerwinkle E. dbNSFPv3.0: a one-stop database of functional predictions and annotations for human nonsynonymous and splice-site SNVs. Hum Mutat. 2016;37: 235-41.

15. Bertram L, McQueen MB, Mullin K, Blacker D, Tanzi RE. Systematic meta-analyses of Alzheimer disease genetic association studies: the AlzGene database. Nat Genet. 2007;39:17-23.

16. Wall DP, Pivovarov R, Tong M, Jung J-Y, Fusaro VA, DeLuca $\mathrm{TF}$, et al. Genotator: a disease-agnostic tool for genetic annotation of disease. BMC Med Genom. 2010;3:50.

17. Li MX, Gui HS, Kwan JSH, Bao SY, Sham PC. A comprehensive framework for prioritizing variants in exome sequencing studies of Mendelian diseases. Nucleic Acids Res. 2012;40:e53 https:// doi.org/10.1093/nar/gkr1257.

18. Franceschini A,Szklarczyk D,Frankild S,Kuhn M,Simonovic M, Roth A, et al. STRINGv9.1: Protein-protein interaction networks, with increased coverage and integration. Nucleic Acids Res. 2013;41:D808-15.

19. Chang CC, Chow CC, Tellier LC, Vattikuti S, Purcell SM, Lee JJ. Second-generation PLINK: rising to the challenge of larger and richer datasets. Gigascience. 2015;4:1-16.

20. MapThin: Thinning your Map Files for Linkage Analyses!. https://www.staff.ncl.ac.uk/richard.howey/mapthin/using.html 
21. Abecasis GR, Cherny SS, Cookson WO, Cardon LR. Merlin-rapid analysis of dense genetic maps using sparse gene flow trees. Nat Genet. 2002;30:97-101.

22. Thiele H, Nurnberg P. HaploPainter: a tool for drawing pedigrees with complex haplotypes. Bioinformatics. 2004;21:1730-2.

23. Egeland T, Pinto N, Vigeland MD. A general approach to power calculation for relationship testing. Forensic Sci Int Genet. 2014;9:186-90.

24. Guerreiro R, Sassi C, Gibbs J, Edsall C, Hernandez D, Brown K et al. A comprehensive assessment of benign genetic variability for neurodegenerative disorders. bioRxiv. 2018. http://biorxiv.org/ content/early/2018/02/23/270686.abstract.

25. Schlöndorff J, Blobel CP. Metalloprotease-disintegrins: modular proteins capable of promoting cell-cell interactions and triggering signals by protein-ectodomain shedding. J Cell Sci. 1999;112(Pt 2):3603-17.

26. Gonzales PE, Solomon A, Miller AB, Leesnitzer MA, Sagi I, Milla ME. Inhibition of the tumor necrosis factor-alphaconverting enzyme by its pro domain. J Biol Chem. 2004;279:31638-45.

27. Peiretti F, Canault M, Deprez-Beauclair P, Berthet V, Bonardo B, Juhan-Vague I, et al. Intracellular maturation and transport of tumor necrosis factor alpha converting enzyme. Exp Cell Res. 2003;285:278-85.

28. Schlöndorff J, Becherer JD, Blobel CP. Intracellular maturation and localization of the tumour necrosis factor alpha convertase (TACE). Biochem J. 2000;347(Pt 1):131-8.

29. Ohtsu H, Dempsey PJ, Frank GD, Brailoiu E, Higuchi S, Suzuki $\mathrm{H}$, et al. ADAM17 mediates epidermal growth factor receptor transactivation and vascular smooth muscle cell hypertrophy induced by angiotensin II. Arterioscler Thromb Vasc Biol. 2006;26:e133-7. https://doi.org/10.1161/01.ATV.0000236203. 90331.d0.

30. Shaul YD, Yuan B, Thiru P, Nutter-Upham A, McCallum S, Lanzkron C, et al. MERAV: a tool for comparing gene expression across human tissues and cell types. Nucleic Acids Res. 2015;44: gkv1337.

31. Suh J, Choi S, Romano DM, Gannon MA, Lesinski AN, Kim DY, et al. ADAM10 missense mutations potentiate $\beta$-amyloid accumulation by impairing prodomain chaperone function. Neuron. 2013;80:385-401.

32. Huang Y-WA, Zhou B, Wernig M, Sudhof TC. ApoE2, ApoE3, and ApoE4 differentially stimulate APP transcription and A $\beta$ secretion. Cell. 2017;168:427-441.e21.

33. von Rotz RC. The APP intracellular domain forms nuclear multiprotein complexes and regulates the transcription of its own precursor. J Cell Sci. 2004;117:4435-48.

34. Bukhari H, Glotzbach A, Kolbe K, Leonhardt G, Loosse C, Müller T. Small things matter: Implications of APP intracellular domain AICD nuclear signaling in the progression and pathogenesis of Alzheimer's disease. Prog Neurobiol. 2017;156:189213.

35. Rossner S, Ueberham U, Schliebs R, Perez-Polo JR, Bigl V. p75 and TrkA receptor signaling independently regulate amyloid precursor protein mRNA expression, isoform composition, and protein secretion in PC12 cells. J Neurochem. 1998;71:757-66.

36. Weskamp G, Schlöndorff J, Lum L, Becherer JD, Kim TW, Saftig $\mathrm{P}$, et al. Evidence for a critical role of the tumor necrosis factor $\alpha$ convertase (TACE) in ectodomain shedding of the p75 neurotrophin receptor (p75NTR). J Biol Chem. 2004;279:4241-9.

37. Amara FM, Junaid a, Clough RR, Liang B. TGF-beta(1), regulation of alzheimer amyloid precursor protein mRNA expression in a normal human astrocyte cell line: mRNA stabilization. Brain Res Mol Brain Res. 1999;71:42-9.

38. Kawasaki K, Freimuth J, Meyer DS, Lee MM, TochimotoOkamoto A, Benzinou M, et al. Genetic variants of Adam17 differentially regulate TGF signaling to modify vascular pathology in mice and humans. Proc Natl Acad Sci USA. 2014;111:7723-8.

39. Rybnikova E, Gluschenko T, Galeeva A, Tulkova E, Nalivaeva NN, Makova NZ, et al. Differential expression of ADAM15 and ADAM17 metalloproteases in the rat brain after severe hypobaric hypoxia and hypoxic preconditioning. Neurosci Res. 2012;72: 364-73.

40. Tian Y, Crump CJ, Li Y-M. Dual role of alpha-secretase cleavage in the regulation of gamma-secretase activity for amyloid production. J Biol Chem. 2010;285:32549-56.

41. Pietri M, Dakowski C, Hannaoui S, Alleaume-Butaux A, Hernandez-Rapp J, Ragagnin A, et al. PDK1 decreases TACEmediated alpha-secretase activity and promotes disease progression in prion and Alzheimer's diseases. Nat Med. 2013;19: 1124-31.

42. Pietri M, Dakowski C, Hannaoui S, Alleaume-Butaux A, Hernandez-Rapp J, Ragagnin A, et al. PDK1 decreases TACEmediated??-secretase activity and promotes disease progression in prion and Alzheimer's diseases. Nat Med. 2013;19:1124-31.

43. Miyazaki T, Ikeda K, Horie-Inoue K, Inoue S. Amyloid precursor protein regulates migration and metalloproteinase gene expression in prostate cancer cells. Biochem Biophys Res Commun. 2014;452:828-33.

44. Feuerbach D, Schindler P, Barske C, Joller S, Beng-Louka E, Worringer KA, et al. ADAM17 is the main sheddase for the generation of human triggering receptor expressed in myeloid cells (hTREM2) ectodomain and cleaves TREM2 after Histidine 157. Neurosci Lett. 2017;660:109-14.

45. Drmanac R, Sparks AB, Callow MJ, Halpern AL, Burns NL, Kermani BG et al. Human genome sequencing using unchained base reads on self-assembling DNA nanoarrays. Science (80-) 2010; 327: 78-81. 